\title{
Traceability of Biologics in The Netherlands: An Analysis of Information-Recording Systems in Clinical Practice and Spontaneous ADR Reports
}

\author{
Kevin Klein ${ }^{1,2,3}$ • Joep H. G. Scholl ${ }^{4}$ Niels S. Vermeer ${ }^{1,5}$ • André W. Broekmans ${ }^{2}$ • \\ Eugène P. Van Puijenbroek ${ }^{4}$ Marie L. De Bruin ${ }^{1,5} \cdot$ Pieter Stolk $^{1,2,3}$
}

Published online: 30 December 2015

(C) The Author(s) 2015. This article is published with open access at Springerlink.com

\begin{abstract}
Introduction and Objective Pharmacovigilance requirements for biologics mandate that EU Member States shall ensure that any biologic that is the subject of a suspected adverse drug reaction (ADR) is identifiable by brand name and batch number. Recent studies showed that brand name identification is well established, whereas batch numbers are (still) poorly reported. We evaluated information-recording systems and practices in the Dutch hospital setting to identify determinants for brand name and batch number recording as well as success factors and bottlenecks for traceability.

Methods We surveyed Dutch hospital pharmacists with an online questionnaire on systems and practices in hospitals for recording brand names and batch numbers. Additionally, we performed an analysis of the traceability of recombinant biologics in spontaneous ADR reports
\end{abstract}

Electronic supplementary material The online version of this article (doi:10.1007/s40264-015-0383-8) contains supplementary material, which is available to authorized users.

Marie L. De Bruin

M.L.deBruin@uu.nl

Kevin Klein

k.klein@escher-project.org

1 Division of Pharmacoepidemiology and Clinical Pharmacology, Utrecht Institute for Pharmaceutical Sciences (UIPS), Utrecht University, Universiteitsweg 99, 3584 CG Utrecht, The Netherlands

2 TI Pharma Escher, Leiden, The Netherlands

3 Exon Consultancy, Amsterdam, The Netherlands

4 The Netherlands Pharmacovigilance Centre Lareb, Den Bosch, The Netherlands

5 Medicines Evaluation Board (MEB), Utrecht, The Netherlands (received between 2009 and 2014) from the Netherlands Pharmacovigilance Centre Lareb.

Results The survey showed that brand names are not routinely recorded in the clinical practice of Dutch hospitals, whereas batch numbers are poorly recorded. Seventysix percent of the 1523 ADR reports for recombinant biologics had a traceable brand name whereas $5 \%$ of these reports contained a batch number. The results suggest a possible relationship between the availability of brand and batch number information in clinical practice and the inclusion of this information in ADR reports for biologics. Conclusion The limited traceability of brand names and batch numbers in ADR reports may be primarily caused by the shortcomings in the recording of information in clinical practice. We recommend efforts to improve informationrecording systems as a first step to improve the traceability of biologics in ADR reporting.

\section{Key Points}

Brand names are not routinely recorded in Dutch clinical practice for medicinal products dispensed and administered to patients; moreover, batch numbers are poorly recorded overall.

Product and batch information recording in clinical practice for biologics is necessary for the retrieval of detailed exposure information in case of an adverse drug reaction.

Shortcomings in the recording and tracing of exposure information in clinical practice may be associated with the limited traceability of biologics in ADR databases such as EudraVigilance. 


\section{Introduction}

Biologics differ from small molecule medicines in their highly complex structures and sensitivity to changes in the manufacturing process $[1,2]$. These distinctive properties for biologics have consequences for the authorisation of follow-on biologics (biosimilars), but also for maintaining constant product quality and safety profiles throughout the lifecycle of a biologic [3, 4]. Manufacturing variability between and within products over time may result in previously unobserved adverse drug reactions (ADRs) [5, 6]. For this reason, biologics are subject to specific pharmacovigilance requirements, which mandate that EU Member States take all appropriate measures to ensure that any biologic that is the subject of a suspected ADR report needs to be identifiable by brand name and batch number [7]. Adequate availability of this exposure information is necessary to timely link an emerging product safety issue to the correct product and batch [8].

In the EU, national competent authorities (NCAs) for pharmacovigilance and marketing authorisation holders (MAHs) collect reports of suspected ADRs at the national level, which are then aggregated in EudraVigilance, the EU ADR database that is maintained by the European Medicines Agency (EMA), to support signal detection and analysis. A recent study by Cutroneo et al. in the Italian ADR database showed that for $94.8 \%$ of the entries for biologics, identifiable brand names were reported [9]. For ADR reports for biologics for which a biosimilar was on the market, this number was even higher $(98.7 \%)$. A study by Vermeer et al. in EudraVigilance showed that $96.2 \%$ of the entries for suspected biologics were identifiable by brand name [10]. In contrast, both the Cutroneo et al. and the Vermeer et al. analyses showed that batch number reporting was poor (8.6 and $21.1 \%$, respectively).

Previous studies quantifying the traceability and identifiability of biologics in spontaneous reporting databases concluded that the traceability of biologics is not adequately ensured and encouraged stakeholders to undertake efforts to improve the current situation. However, factors that may contribute to the lack of traceability have not been studied in detail yet. In the present study, we therefore aimed to identify determinants for brand name and batch number recording as well as critical success factors and bottlenecks for ensuring the traceability of biologics in ADR reporting. We used the Dutch hospital setting as a case to evaluate the information-recording practices, as the hospital setting plays a central role in the distribution process of most biologics, and evaluated influencing factors for the reporting of brand names and/or batch numbers in ADR reports for biologics.

\section{Methods}

We approached this study with a mixed quantitative and qualitative method. We first evaluated the informationrecording systems and practices that are in place in the Dutch hospital setting for brand name and batch number recording, by surveying hospital pharmacists about information-recording systems and practices within their hospital. Secondly, we performed a comprehensive analysis of the traceability of biologics in spontaneous ADR reports from the Dutch ADR database at the Netherlands Pharmacovigilance Centre Lareb, comparing ADR reports originating from the hospital setting with other report sources.

\subsection{Assessment of In-Hospital Information- Recording Systems and Practices}

\subsubsection{An Overview of the Hospital Setting}

In general, three processes can be distinguished within the hospital setting [11-13].

(i) The drug distribution process. The drug distribution process starts with the distribution of the drug from the manufacturing plant to the wholesaler and, next, to the hospital pharmacy.

(ii) The healthcare process. When a drug has been delivered to the hospital pharmacy, it enters the healthcare process, which starts with the physician prescribing a drug, the hospital pharmacist dispensing the drug, a physician or nurse administering the drug and the patient receiving the drug and being monitored by the physician.

(iii) The information-recording process. At different steps in the healthcare (and distribution) process, information-recording systems may be used to record information about a medicinal product; for example, by scanning barcodes on the outer package.

In the Netherlands, different types of informationrecording systems can be identified: hospital pharmacy information systems (HPIS) at the dispensing phase, electronic medication administration records (eMAR) at the administration phase and the electronic health records (EHR) of the patient, which capture a variety of administrative and medical data about the patient. ${ }^{1}$ These information-recording systems may or may not exchange information with each other or be incorporated in one

\footnotetext{
1 Another system that is in place in Dutch hospitals is the computerised physician order entry (CPOE) used for prescribing a drug. This system is not further discussed in this study because it captures what is prescribed and not which drug has actually been dispensed/administered to the patient.
} 
integral system [14]. Additionally, hospital pharmacists in the Netherlands keep a record within the pharmacy of medicinal products that are prepared for administration to a specific patient. This record, called the compounding protocol, may provide another opportunity to record and trace back medicinal product information (at least for the biologics that are prepared for administration in the hospital pharmacy) [15].

\subsubsection{Survey on Information-Recording Systems and Practices in the Hospital Setting}

The overview described in the previous section provided the framework for a survey among Dutch hospital pharmacists. The survey focused on systems and practices in hospitals for the recording and the availability of medicinal product information, such as brand names and batch numbers (for biologics in particular). The survey was conducted with an online questionnaire that was sent to all members of the Netherlands Association of Hospital Pharmacies representing 93 hospitals in the Netherlands (see electronic supplementary material 1 ). The questionnaire consisted of three sections with a total of 36 questions, comprising a mix of multiple choice and open questions. The questionnaire started with questions about the information-recording systems that are in place in Dutch hospitals, followed by questions about the experience of the hospital pharmacists with ADR reporting for biologics and, finally, the questionnaire asked for personal recommendations on how to improve the traceability of biologics in clinical practice. The last section consisted of open questions that could be responded to voluntarily.

\subsection{Analysis of ADR Data from The Netherlands Pharmacovigilance Centre Lareb}

\subsubsection{Study Sample}

For the analysis of ADR reports in the Netherlands, we used a pre-defined list of recombinant biologics to select relevant reports. The list of recombinant biologics was created based on an extraction of all centrally authorised medicinal products found in the EMA database of European Public Assessment Reports (EPARs) on November 30,2014 . A product was included on our list if recombinant technology or cell lines were mentioned for the description of the manufacturing process in Section 2 of the Summary of Product Characteristics (SmPC) entitled "Qualitative and quantitative composition". Seven recombinant biologics (four somatropins, one epoetin alfa, one insulin human and one filgrastim) that were authorised prior to 1995 were also added to the list because they are still commonly used in clinical practice in the Netherlands.
Recombinant vaccines were not included in this study. The 146 recombinant biologics used for this study were categorised in 11 mechanistic product classes (see electronic supplementary material 2).

Based on ATC codes, we extracted spontaneous reports of suspected ADRs for the products on our sample list received between January 01, 2009 and December 31, 2014 from the database of The Netherlands Pharmacovigilance Centre Lareb. For this analysis, we only included spontaneous ADR reports that have been directly reported to the Netherlands Pharmacovigilance Centre Lareb. PL/SQL Developer Version 8.0.41514 was used for the extraction of ADR data from the Dutch ADR database.

\subsubsection{Data Classification and Outcome Analysis}

Brand name and batch number entry fields were reviewed to determine whether the brand name was identifiable and whether reported batch numbers referred to valid entries (e.g. to exclude false entries such as "unknown" or "?"). The traceability of biologics in ADR reports was assessed based on the number of reports that included a brand name and batch number. Data were stratified by reporter type, the year in which the report was received and product class. All values are presented as absolute numbers and proportions.

\section{Results}

\subsection{Assessment of In-Hospital Information- Recording Systems and Practices}

\subsubsection{Evaluation of the Information-Recording Systems and Practices in the Hospital Setting}

The online survey among hospital pharmacists had a response rate of $37 \%$ (34 out of 93 hospitals). Twentyseven out of 34 (79\%) hospital pharmacists responded that brand names are routinely recorded in the HPIS, which is only accessible to hospital pharmacists and technicians as indicated by $33(97 \%)$ respondents and to a smaller extent to physicians as indicated by $14(41 \%)$ respondents (Table 1). Twenty-five (74\%) hospital pharmacists indicated that eMAR is in place in their hospital. Fifteen out of these $25(60 \%)$ hospitals with eMAR responded that brand names are routinely recorded in eMAR during the administration phase. Twenty-two out of all $34(65 \%)$ respondents indicated that brand names are routinely recorded in the EHR. A total of seven (21\%) hospitals do not record brand names routinely in any of the available informationrecording systems that are in place. None of the respondents indicated that batch numbers are routinely recorded in the HPIS, eMAR or EHR (Table 1). 
Table 1 Brand name and batch number recording in different information-recording systems in the Dutch hospital setting

\begin{tabular}{llll}
\hline $\begin{array}{l}\text { Information-recording } \\
\text { system }\end{array}$ & $\begin{array}{l}\text { Respondents indicating system } \\
\text { in place in hospital }[n]\end{array}$ & $\begin{array}{l}\text { Brand name } \\
\text { recording }[n(\%)]\end{array}$ & $\begin{array}{l}\text { Batch number } \\
\text { recording }[n(\%)]\end{array}$ \\
\hline HPIS & 34 & $27(79)$ & $0(0)$ \\
eMAR & 25 & $15(60)$ & $0(0)$ \\
EHR & 34 & $22(65)$ & $0(0)$ \\
\hline
\end{tabular}

HPIS Hospital pharmacy information system, eMAR electronic medication administration record, EHR electronic health record

In addition to the information-recording systems, 23 $(68 \%)$ respondents indicated that brand names are routinely recorded in the compounding protocol, whereas all $34(100 \%)$ respondents indicated that batch numbers are routinely recorded in the compounding protocol. The information in the compounding protocol is, however, only available to hospital pharmacists and technicians as indicated by all respondents.

For comparison, a post-hoc exploratory survey outside the hospital setting among 56 community pharmacists indicated that $51(91 \%)$ community pharmacists routinely record brand names in their community pharmacy information system for medicinal products dispensed to patients.

\subsubsection{Bottlenecks and Potential Solutions for the Traceability of Biologics}

The open questions about the opinions of hospital pharmacists about the main bottlenecks and possible solutions for the traceability of biologics in clinical practice resulted in 26 responses. The main reported bottleneck that hampers the traceability of biologics in clinical practice is that batch number information is not encoded in barcodes and can therefore not automatically be recorded in informationrecording systems, as mentioned by 19 (73\%) hospital pharmacists. Ten (38\%) hospital pharmacists mentioned that the barcodes that are present are often not available on the single unit dose and can therefore not be scanned for information-recording purposes. Eight (31\%) hospital pharmacists indicated that due to the lack of batch number entry fields in current information-recording systems, it is not possible to (manually) record batch numbers.

The hospital pharmacists who provided potential solutions were fairly in agreement; 19 out of 26 (73\%) respondents indicated that barcodes with encoded batch numbers or a similar method could offer a robust solution by facilitating (automatic) recording of batch number information in the information-recording systems. Moreover, 10 (38\%) hospital pharmacists specifically called for the provision of barcodes on the single unit dose, since this is the packaging that needs to be scanned during the dispensing phase in HPIS and during the administration phase in eMAR.

\section{1 .3 Awareness}

The results of the hospital pharmacy survey also showed a limited awareness about the requirement to report the brand name and the batch number in ADR reports for biologics. Six out of $34(18 \%)$ hospital pharmacists indicated that they are unaware of the need to include brand names in ADR reports for biologics, whereas four (12\%) hospital pharmacists indicated that they do not see the purpose for reporting the brand names. When it comes to batch number reporting, $14(41 \%)$ hospital pharmacists responded that they were not aware that they should include batch numbers for ADR reports for biologics, whereas two $(6 \%)$ respondents indicated that they do not see the need to include batch numbers in ADR reports for biologics.

\subsection{Analysis of ADR Data from The Netherlands Pharmacovigilance Centre Lareb}

The Netherlands Pharmacovigilance Centre Lareb received 43,907 spontaneous ADR reports in the study period, in which 1523 recombinant biologics from our sample list were reported as suspected drugs. For $76 \%$ of these biologics that were the subject of a suspected ADR report, a brand name was identifiable, whereas $5 \%$ of the ADR reports contained a batch number.

\subsubsection{Reporter Type Analysis}

Physicians and nurses accounted for the majority (57 \%) of the ADR reports from our study sample, whereas hospital pharmacists accounted for $5 \%$ of the entries. The remaining $38 \%$ of the ADR reports were received from community pharmacists $(16 \%)$, patients $(15 \%)$, general practitioners $(5 \%)$ and unclassified (2\%).

As shown in Table 2, community pharmacists are most likely to report a brand name ( $96 \%$ ), followed by patients $(87 \%)$, general practitioners (86\%), hospital pharmacists $(69 \%)$ and physicians and nurses (68\%). However, when it comes to batch number reporting, hospital pharmacists are most likely to report batch numbers (36\%), whereas physicians and nurses are least likely to report a batch number $(3 \%)$. 
Table 2 Brand name and batch number reporting by reporter type of adverse drug reaction (ADR) reports of recombinant biologics received by the Netherlands Pharmacovigilance Centre Lareb (2009-2014)

\begin{tabular}{lccc}
\hline Reporter type & ADRs reported $\left[n(\%)^{\mathrm{a}}\right]$ & Brand names reported $\left[n(\%)^{\mathrm{b}}\right]$ & Batch numbers reported $\left[n(\%)^{\mathrm{b}}\right]$ \\
\hline Hospital: physician/nurse & $866(57)$ & $587(68)$ & $13(2)$ \\
Hospital: pharmacist & $78(5)$ & $54(69)$ & $28(36)$ \\
Community: GP & $78(5)$ & $67(86)$ & $2(3)$ \\
Community: pharmacist & $239(16)$ & $229(96)$ & $17(7)$ \\
Patient & $223(15)$ & $193(87)$ & $3(5)$ \\
Unclassified & $39(2)$ & $1152(76)$ & $74(5)$ \\
Total & $1523(100)$ & &
\end{tabular}

$G P$ general practitioner (community setting)

a These percentages are proportions of the total of 1523 ADR reports

b These percentages represent the proportion of the total number of ADRs reported by the reporter type in the respective row

\subsubsection{Reporting Over Time}

When the ADR reporting data were stratified by year, no visible increase over time in brand name or batch number reporting and no change after the introduction of the new pharmacovigilance legislation was observed (Fig. 1). ${ }^{2}$

\subsubsection{Product Class Analysis}

For each product class, we analysed the total numbers of ADR reports and the percentages of brand name and batch number identification (Table 3). More than half of the ADR reports for recombinant biologics in the Dutch ADR database concerned monoclonal antibodies. We also observed class-specific differences for brand name reporting. Batch number reporting varied from $0 \%$ to a maximum of $10 \%$ between different product classes.

The product class other consisted of recombinant proteins such as teriparatide, for example. Only a small number of ADR reports $(n=37)$ were received for biologics for which a biosimilar is on the market in the Netherlands (somatropin, epoetin alfa and filgrastim), which did not allow for an analysis of the traceability of the brand name for these specific product classes.

\section{Discussion}

\subsection{Main Findings}

Our analysis showed that brand names are not routinely recorded in the clinical practice of Dutch hospitals,

\footnotetext{
2 The pharmacovigilance legislation was implemented in the Netherlands in January 2013 (https://zoek.officielebekendmakingen.nl/stb2013-21.html [in Dutch]).
}

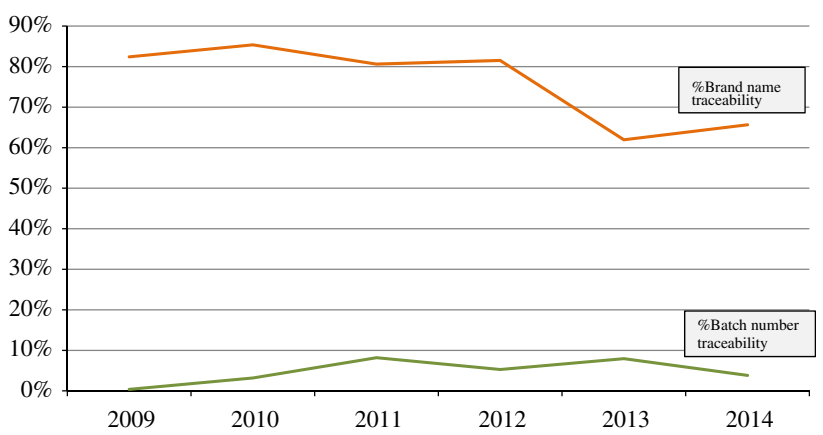

Fig. 1 Brand name and batch number reporting over time: traceability of spontaneous adverse drug reaction (ADR) reports of recombinant biologics received by the Netherlands Pharmacovigilance Centre Lareb (2009-2014)

whereas batch numbers are poorly recorded. This prevents adequate traceability of brand and batch information for the purpose of ADR reporting. The results from the survey together with the ADR data analysis suggest a possible relationship between the availability of (and thus the ability to trace) brand name and batch number information in clinical practice and the inclusion of this information in ADR reports for biologics. The main bottlenecks for ensuring the traceability of biologics in ADR reporting to the level of the batch number are the absence of adequate information-recording systems that allow tracing of this information and missing information on the product or single unit dose.

\subsection{Brand Name Traceability}

As the findings from our study indicated, brand names are not routinely recorded in the information-recording systems in the hospital setting. This could explain why healthcare professionals from the hospital setting including 
Table 3 Brand name and batch number reporting of recombinant biologics by product class in adverse drug reaction (ADR) reports received by the Netherlands Pharmacovigilance Centre Lareb (2009-2014)

\begin{tabular}{lccc}
\hline Product class & $\begin{array}{l}\text { ADRs } \\
\text { reported }[n]\end{array}$ & $\begin{array}{l}\text { Brand names } \\
\text { reported }[n(\%)]\end{array}$ & $\begin{array}{l}\text { Batch numbers } \\
\text { reported }[n(\%)]\end{array}$ \\
\hline Somatropins & 4 & $3(75)$ & $0(0)$ \\
Epoetins & 43 & $40(93)$ & $0(0)$ \\
Filgrastims & 19 & $17(89)$ & $1(5)$ \\
Follitropins & 21 & $21(100)$ & $1(5)$ \\
Monoclonal antibodies & 797 & $536(67)$ & $45(6)$ \\
Insulins & 180 & $164(91)$ & $18(10)$ \\
Interferons & 51 & $45(88)$ & $3(6)$ \\
Antihaemophilic factors & 52 & $52(100)$ & $1(2)$ \\
Fusion proteins & 232 & $178(77)$ & $5(2)$ \\
Enzymes & 2 & $1(50)$ & $0(0)$ \\
Other & 122 & $95(78)$ & $0(0)$ \\
Total & 1523 & $1152(76)$ & $74(5)$ \\
\hline
\end{tabular}

hospital pharmacists include brand names in ADR reports in only $68-69 \%$ of the cases. This is in contrast to the percentage of brand name reporting for reporter types outside the hospital setting, for example the community pharmacists, who include brand names in $96 \%$ of the cases. The post-hoc exploratory survey among community pharmacists referred to in the results section supported these findings: $91 \%$ of the community pharmacists indicated that brand names are routinely recorded in the community pharmacy information system for medicinal products dispensed to patients.

This may also explain the difference between product classes observed in Table 3. An ADR report for the monoclonal antibodies, which in the Netherlands is mostly dispensed in the hospital setting, has identifiable brand names in $67 \%$ of the cases. However, ADR reports for insulins, which are mostly dispensed in the community setting, have identifiable brand names in $91 \%$ of the cases. An explanation for why the systematic recoding of brand names is not always common practice in the hospital setting (as compared with the community setting) could be the Dutch reimbursement system for medicines. Hospitals are financially compensated according to agreed budget allocations for a specific type of treatment; therefore, brand names are not necessarily required for invoicing purposes [16]. However, reimbursement in the community pharmacy setting is based on a reimbursement system for specific brands, requiring a comprehensive system to record which specific brand of the medicinal product has been dispensed [17].

\subsection{Batch Number Traceability}

A possible association between the availability of medicinal product information and the inclusion of this information in ADR reports is also observed for batch numbers. As shown by the results from the pharmacist survey, batch numbers are not routinely recorded in hospital informationrecording systems. This means that the batch number information is lost and cannot be traced after the (primary) package of the medicinal product has been discarded [18]. However, our study also found that batch numbers are routinely recorded in the compounding protocol for medicinal products that are prepared for administration in the hospital pharmacy, which supports the findings from a previous study [15]. Although this only concerns biologics that are compounded in the hospital pharmacy, it was observed that hospital pharmacists (who are the only healthcare professionals with direct access to this information) are most likely to include batch numbers in ADR reports $(36 \%)$ compared with other reporter types. We believe that the majority of ADR reports from hospital pharmacists with identifiable batch numbers concern biologics that are prepared in the hospital pharmacy, since these reports mainly concern biologics that are supplied in formulations that require an additional preparatory step before administration to the patient (e.g. powder form).

\subsection{Limitations}

There are several limitations that need to be considered when interpreting the results of this study. It should be noted that there is only a limited number of ADR reports available for the sample list of recombinant biologics included in this analysis, which does not allow for extensive data analysis. In addition, we only included recombinant biologics in the ADR data analysis. However, we have no reasons to assume that the ADR reporting quality would have strongly diverged when including data for non-recombinant biologics as well.

There are certain limitations that apply to online surveys like the one used in this study; for example, some questions may be misinterpreted or filled in incorrectly due to a lack 
of understanding or time constraints. We offered several open questions and explanatory text fields to allow responders to provide additional information, explain their answers or to indicate if they do not know the answer to a question. However, when looking at the answers we believe that there was a clear pattern emerging from the respondents. We have no reason to assume that there was a non-response bias.

Although the generalisability of the results of this study to European practice is potentially limited considering the expected heterogeneity in national policies and guidelines, we believe that the Netherlands provides a good case study to demonstrate the importance of information-recording systems in current pharmacovigilance activities and the challenges and bottlenecks encountered. It would, however, be useful to conduct comparable analyses in other EU Member States. A detailed ADR data analysis in other Member States could provide new insights into information-recording systems and practices, and identify more bottlenecks and success factors for the traceability of biologics.

\subsection{Technical Solutions to Improve Traceability in Clinical Practice}

The online questionnaire among hospital pharmacists showed that the majority of hospital pharmacists expressed the need for barcodes with encoded batch number information on the single unit dose to improve the traceability of biologics. This would allow electronic recording of medicinal product information (such as batch numbers) of individual packages throughout the supply chain; for example, bedside scanning at the administration site. The implementation of a new 'standard' barcode may impose challenges to the current ICT infrastructure of stakeholders involved and may require a change of practice. Finding synergies with other initiatives that may benefit from a new barcode may therefore be critical. For example, in a full track-and-trace system, a barcode containing batch number information applied to the single unit dose (often the primary package) may provide opportunity for synergies with other important topics, such as improvement of the efficiency of batch recalls, optimisation of inventory management in pharmacies, increased transparency of the distribution chain and the reduction of medication errors. The GS1 Data Matrix is a two-dimensional barcode that allows batch number encoding and will be mandatory by 2018 as part of the Falsified Medicines Directive. The Directive, however, states that the GS1 Data Matrix, in which the inclusion of the batch number will be mandatory, will only be applied to the outer package (secondary packaging) [19-21].

\subsection{Awareness}

Our study showed that a lack of awareness still could play a role in the poor traceability of biologics in ADR reporting, which is a frequently stated cause in the literature [22]. However, as our analysis in the Netherlands shows, apart from raising awareness, improvements in the area of healthcare systems and practices for recording and tracing medicinal product information are fundamentally required as this could be the root causes for the poor traceability of biologics in ADR reporting and therefore needs to be addressed in tandem.

\section{Conclusion}

In conclusion, this study showed that, in the Netherlands, brand names are not routinely recorded in clinical practice and batch numbers are poorly recorded. These findings suggest that the limited traceability of brand names and batch numbers in ADR reports for biologics may be primarily caused by the shortcomings in the recording and tracing of information in clinical practice. Although a lack of awareness may contribute to this issue, efforts to address the systems for information recording and sharing in clinical practice are needed. Furthermore, the implementation of a barcode on the single unit dose that contains both brand name and batch number information may be needed as a first step to improve the traceability of (biological) medicinal products in clinical practice. Concerted actions such as these can help to build a system that is able to support the achievement of the public health objectives that are the reason for current regulations for the pharmacovigilance of biologics in Europe. From a broader perspective, the findings presented in this study should also highlight the need for EU policy makers to evaluate national regulatory instruments prior to the implementation of new regulations and to assess how EU regulations can be used at the national level in an appropriate and pragmatic manner.

Acknowledgments The Netherlands Association of Hospital Pharmacists (NVZA) and the Royal Dutch Pharmacists Association (KNMP) are thanked for their support in this study and by providing advice and access to their network.

\section{Compliance with Ethical Standards}

Funding The work of KK, NV, AB, MB and PS on this project was supported by Escher, the TI Pharma Platform for Regulatory Innovation. Escher is an independent platform and receives funding from public and private partners to support their activities. For more information, please visit http://escher.tipharma.com. This Escher research was funded with an unrestricted grant from European 
Biopharmaceutical Enterprises (EBE). JS and EP are employed by the Netherlands Pharmacovigilance Centre Lareb.

Conflict of interest Kevin Klein, Joep H. G. Scholl, Niels S. Vermeer, André W. Broekmans, Eugène P. Van Puijenbroek, Marie L. De Bruin and Pieter Stolk declare that they have no conflicts of interest.

Open Access This article is distributed under the terms of the Creative Commons Attribution-NonCommercial 4.0 International License (http://creativecommons.org/licenses/by-nc/4.0/), which permits any noncommercial use, distribution, and reproduction in any medium, provided you give appropriate credit to the original author(s) and the source, provide a link to the Creative Commons license, and indicate if changes were made.

\section{References}

1. European Commission. What you need to know about biosimilar medicinal products. 18 December 2014. http://ec.europa.eu/Docs Room/documents/8242/attachments/1/translations/en/renditions/pdf. Accessed 23 Oct 2015.

2. Sharma B. Immunogenicity of therapeutic proteins. Part 3: impact of manufacturing changes. Biotechnol Adv. 2007;25:325-31.

3. Chirino AJ, Mire-Sluis A. Characterizing biological products and assessing comparability following manufacturing changes. Nat Biotechnol. 2004;22:1383-91.

4. Schiestl M, Stangler T, Torella C, Čepeljnik T, Toll H, Grau R. Acceptable changes in quality attributes of glycosylated biopharmaceuticals. Nat Biotechnol. 2011;29:310-2.

5. Calvez T, Chambost H, Claeyssens-Donadel S, et al. Recombinant factor VIII products and inhibitor development in previously untreated boys with severe hemophilia A. Blood. 2014; 124:3398-408.

6. Louët S. Lessons from Eprex for biogeneric firms. Nat Biotechnol. 2003;21:956-7.

7. Directive 2010/84/EU of the European Parliament and of the Council of 15 December 2010, amending as regards pharmacovigilance, Directive 2001/83/EC on the Community code relating to medicinal products for human use. http://ec.europa.eu/health/files/eudralex/ vol-1/dir_2010_84/dir_2010_84_en.pdf. Accessed 31 Mar 2015.

8. Calvo B, Zuñiga L. EU's new pharmacovigilance legislation: considerations for biosimilars. Drug Saf. 2014;37:9-18.

9. Cutroneo PM, Isgrò V, Russo A, et al. Safety profile of biological medicines as compared with non-biologicals: an analysis of the italian spontaneous reporting system database. Drug Saf. 2014;37:961-70.

10. Vermeer NS, Straus SMJM, Mantel-Teeuwisse AK, et al. Traceability of biopharmaceuticals in spontaneous reporting systems: a cross-sectional study in the FDA adverse event reporting system (FAERS) and EudraVigilance databases. Drug Saf. 2013;36:617-25.
11. Franklin BD, O'Grady K, Donyai P, Jacklin A, Barber N. The impact of a closed-loop electronic prescribing and administration system on prescribing errors, administration errors and staff time: a before-and-after study. Qual Saf Health Care. 2007;16:279-84.

12. Poon EG, Keohane CA, Yoon CS, et al. Effect of bar-code technology on the safety of medication administration. N Engl J Med. 2010;362:1698-707.

13. Seibert HH, Maddox RR, Flynn EA, Williams CK. Effect of barcode technology with electronic medication administration record on medication accuracy rates. Am J Health Syst Pharm AJHP Off J Am Soc Health Syst Pharm. 2014;71:209-18.

14. Lenderink BW, Egberts TCG. Closing the loop of the medication use process using electronic medication administration registration. Pharm World Sci PWS. 2004;26:185-90.

15. Vermeer NS, Spierings I, Mantel-Teeuwisse AK, et al. Traceability of biologicals: present challenges in pharmacovigilance. Expert Opin Drug Saf. 2015;14:63-72.

16. Busse R, editor. The Netherlands: the diagnose behandeling combinaties. In: Diagnosis-related groups in Europe: moving towards transparency, efficiency and quality in hospitals. European observatory on health systems and policies series. Maidenhead: Open Univ. Press; 2011.

17. Zuidberg C. The pharmaceutical system of the Netherlands. 2010. https://ppri.goeg.at/Downloads/Publications/The\%20pharmaceutical $\% 20$ system $\% 20$ of $\% 20$ the $\% 20$ Netherlands_FINAL.pdf. Accessed 2 July 2015.

18. Directive 2001/83/EC of the European Parliament and of the Council of 6 November 2001 on the Community code relating to medicinal products for human use. http://ec.europa.eu/health/ files/eudralex/vol-1/dir_2001_83_cons/dir2001_83_cons_200812 30_en.pdf. Accessed 3 Apr 2015.

19. Commission Delegated Regulation (EU) of 2.10.2015 supplementing Directive 2001/83/EC of the European Parliament and of the Council by laying down detailed rules for the safety features appearing on the packaging of medicinal products for human use. http://ec.europa.eu/transparency/regdoc/rep/3/2015/EN/3-20156601-EN-F1-1.PDF. Accessed 23 Oct 2015.

20. Directive 2011/62/EU of the European Parliament and of the Council of 8 June 2011 amending directive 2001/83/EC on the community code relating to medicinal products for human use, as regards the prevention of the entry into the legal supply chain of falsified medicinal products. http://ec.europa.eu/health/files/ eudralex/vol-1/dir_2011_62/dir_2011_62_en.pdf. Accessed 3 July 2015.

21. European Commission. Delegated act on the detailed rules for a unique identifier for medicinal products for human use, and its verification. Concept paper submitted for public consultation. 18 November 2011. http://ec.europa.eu/health/files/counterf_par_ trade/safety_2011-11.pdf. Accessed 3 July 2015.

22. Getz KA, Stergiopoulos S, Kaitin KI. Evaluating the completeness and accuracy of MedWatch data. Am J Ther. 2014;21:442-6. 